\title{
MARÍA ÁNGELES RODRÍGUEZ GONZÁLEZ: LENGUAJE DE SIGNOS MADRID: CONFEDERACIÓN NACIONAL DE SORDOS DE ESPAÑA/FUNDACIÓN ONCE. 1992
}

Según las últimas estadísticas, en España hay 120.000 sordomudos. A pesar de los esfuerzos bien intencionados de las autoridades pertinentes, dentro del sistema educativo el sordo no goza de igualdad de oportunidades. El método oral que priva en la educación de los sordos desde $1880 \mathrm{y}$ el deseo de integrar a los niños sordos primero en las aulas y luego en la sociedad hacen caso omiso de sus circunstancias y necesidades especiales; el resultado es que entre un setenta y un ochenta por ciento de estos ciudadanos sólo tienen estudios primarios. Incluso en este nivel, como los niños sordos estudian los mismos libros que sus compañeros oyentes, las diferencias entre la sintaxis del español y el lenguaje de signos español (LSE) únicamente sirven para dificultar el proceso de aprendizaje, ya que los niños sordos no siempre tienen los conocimientos necesarios para interpretar sus libros de texto. Por otra parte, los sordos tampoco comprenden el avance del Telediario que Televisión Española emite en «español signado", que no es lo mismo que el LSE.

Está claro que queda mucho por hacer en este campo y el libro de $\mathrm{M}^{\mathrm{a}}$ Á. Rodríguez, fruto de su tesis doctoral, pone la piedra angular al señalar estos fallos. La autora, hablante «bilingüe» de español y LSE, ha publicado otros estudios científicos sobre el LSE así como cuentos infantiles adapta- 
dos al lenguaje gestual. Pero esta obra traza la historia del lenguaje de signos y de la educación de los sordomudos como preludio a la parte central del libro: el estudio lingüístico del LSE.

$\mathrm{Si}$ el lenguaje de signos es un instrumento de comunicación en la misma medida que los lenguajes orales, de allí se sigue que, aparte de las diferencias obvias, podrán descubrirse los mismos usos y parámetros en el lenguaje de signos y en las lenguas habladas. Aunque esto se cumple en determinados casos - los signos se componen de una serie de elementos que se dan simultáneamente en el tiempo y que tienen su paralelo en los rasgos distintivos del fonema, existen mecanismos para la formación de «compuestos» - [PERA + ARBOL $]=$ peral - , el empleo de signos que podrían considerarse «sinónimos» obedece a preferencias debidas a la edad, la situación geográfica o el registro-, el LSE no es lo mismo que el «español signado» y las diferencias, ya se estudien desde la lingüística o desde el punto de vista de los sordomudos, prueban que el LSE es un lenguaje con su propia gramática. Los fallos en el sistema educativo y en la programación de actividades para integrar a los sordomudos en la sociedad de los oyentes parten de nuestro desconocimiento de los mecanismos del LSE.

$\mathrm{Al}$ reconocer que el LSE es un lenguaje, un sistema de comunicación suficiente para sus usuarios, también hay que aceptar el hecho de que estos usuarios no forman un grupo homogéneo ya que las circunstancias de cada uno se translucen en sus intervenciones. Los hablantes «bilingües» (la familia del sordomudo, sus amigos, profesores, etc.) tienden a emplear signos propios del español, modificando así la estructura del lenguaje gestual, como por ejemplo en el uso de la conjunción subordinante que en oraciones sustantivas donde el LSE prefiere la yuxtaposición. En lo que toca al lenguaje, la comunidad de sordos se divide en tres grupos: 1) los sordos congénitos y los que perdieron la facultad de oír antes de cumplir un año, es decir los que no llegaron a desarrollar el lenguaje oral; 2) los que perdieron la audición entre los dos y los cinco años, durante la etapa de adquisición del lenguaje verbal; y 3 ) aquellos cuya sordera les sobrevino a partir de los seis años, los sordos poslocutivos.

Por otra parte, lo que desde fuera parece ser un solo «lenguaje» es, en realidad, tres códigos distintos. El alfabeto manual es una codificación del alfabeto latino que se signa con la mano derecha. Este proceso es lento y poco práctico, por lo que los sordos limitan su uso al deletreo de palabras nuevas o conceptos para los cuales aún no existe un signo. El empleo del alfabeto manual supone el dominio de la lengua escrita, una codificación de la lengua hablada desconocida por parte de los sordos. En este sentido el alfabeto manual es «una lengua extranjera» para los sordos.

A partir de los primeros meses de vida, el niño sordo empieza a desarrollar su propio lenguaje, un medio para comunicarse con los que le 
rodean: éste es el verdadero lenguaje de signos, que se basa en el movimiento de las manos, acompañado por el movimiento corporal y expresividad facial. El movimiento de los labios y la expresividad facial forman parte del lenguaje de signos y no hay que confundirlo con la lectura de labios, una simple variante del lenguaje oral. Cada signo expresa un concepto que puede encadenarse con otros conceptos para enviar un mensaje, para comunicarse.

Como ya hemos señalado, se sabe que la edad en la que tuvo lugar la pérdida de audición influye en la manera en que una persona emplea el lenguaje gestual, y hemos comprobado esto mismo en los hablantes «bilingües» (los casos de transferencia). Existe, por lo tanto, una tercera variante, el «español signado,» que se sirve de los signos gestuales encadenados de acuerdo con una sintaxis española "simplificada.» El español signado es el sistema que emplean los sordos postlocutivos para comunicarse con aquellos oyentes que poseen algunos conocimientos del lenguaje gestual; también es el lenguaje que se emplea en el avance del Telediario que los mismos sordos afirman no entender. Aunque el LSE y el español signado ocupan los extremos de un continuum y, por lo tanto, no son lenguas distintas, el español signado es ajeno a los sordos que perdieron la audición antes de aprender a hablar. No obstante, el español signado sería una herramienta de gran valor en la llamada educación bimodal, según la cual los niños sordos reciben clases de profesores sordos en lenguaje gestual y de profesores «bilingües» en español signado; de esta manera los niños serán «bilingües.» Desgraciadamente la educación bimodal todavía no se ha introducido en España.

La historia del lenguaje gestual y la educación de los sordomudos es más antigua de lo que cabría pensar. M. ${ }^{a}$ Á. Rodríguez recoge unas notas de la Antigüedad antes de estudiar la evolución de la enseñanza de los sordos. En griego $\lambda$ ó ${ }^{\circ} \zeta$ significaba tanto «palabra» como «pensamiento», mientras que koфó Desde esta perspectiva no es sorprendente que el código justiniano no concediera todos los derechos a los sordomudos. Aunque también hay citas de casos excepcionales de sordos que aprendieron a leer y escribir, las notas más positivas atestiguan el hecho de que los sordos tenían su propio medio de comunicación gestual (S. Agustín, sg. IV) y la expresividad de sus gestos (L. da Vinci, sg. XVI).

A Fray Pedro Ponce de León, un monje benedictino de San Salvador de Oña, del siglo XVI, se le considera el primer maestro de sordos y se le atribuye el alfabeto manual. Aunque no tenemos noticias concretas sobre su método, se cree que primeramente enseñaba las letras para despúes relacionar palabras y objetos; a continuación demostraba la pronunciación de las letras, lo que permitía a sus alumnos leer los labios de los demás así como articular sus propios mensajes. Fray Pedro y los demás religiosos que se dedicaron a esta labor limitaron su enseñanza a hijos de nobles en su 
lugar de residencia, de modo que no aprovechó a la población en general. Sin embargo, los extranjeros que vinieron a la Corte española y conocieron a estos sordomudos llevaron noticias del hecho a Inglaterra e Italia.

En la Francia del siglo XVIII el abad de l'Epée basó las primeras enseñanzas en el lenguaje gestual que los mismos sordos habían desarrollado, al cual añadió otros signos. De l'Epée se dio cuenta de que un conjunto de letras (el alfabeto manual) en principio no tenía significado alguno para los sordos, mientras que si éstos lograban relacionar una palabra escrita con el signo gestual correspondiente existía la certeza de que el alumno había entendido. Pero el abate no comprendió la importancia ni las posibilidades del lenguaje gestual y lo utilizó como un mero útil para la enseñanza del francés, el mismo principio que rige la actual educación bimodal. Conviene resaltar además el hecho de que de l'Epée no se limitó a la enseñanza de nobles sino que impartía lecciones a un grupo de unas treinta personas con distintos niveles de conocimientos.

Pronto aparecieron los detractores del uso del lenguaje gestual en el aula. Se argumentaba que el lenguaje de signos no era un verdadero lenguaje y que no servía para incorporar a los sordomudos en la sociedad de los oyentes. El Congreso Internacional de Maestros de Sordomudos celebrado en Milán en 1880 propugnó esta doctrina, que sigue vigente hoy. Por muy loable que sea intentar integrar a los minusválidos en la sociedad, hay que reconocer que los métodos al uso han fracasado. Un siglo más tarde son elocuentes las palabras de la presidenta de la asociación de sordos de Valladolid: «El sordo no duda del esfuerzo y buena voluntad de sus educadores, pero se está queriendo hacer del niño sordo un adulto oyente, cuando hay que hacer del niño sordo un adulto sordo con posibilidades de acercamiento al oyente.»

El presente estudio brinda abundante información a un mundo desinformado. Proporciona datos generales referentes a la sordera y al lenguaje gestual así como un capítulo dedicado a la historia de la enseñanza de los sordomudos. Sin embargo, la parte central de este libro es un estudio del lenguaje gestual como lenguaje propio, diferente de cualquier otro lenguaje en la misma medida que el español es distinto del francés y no en la medida en que la palabra escrita de un idioma dado difiere de la lengua hablada. Por lo tanto, si se tratara de una verdadera lengua, esperaríamos descubrir en el LSE los mismos rasgos que caracterizan cualquier otro lenguaje humano, hasta donde esto sea posible. He aquí la finalidad del presente estudio.

En el lenguaje gestual la Querología ocupa el lugar de la Fonología, ya que los haces de rasgos distintivos en el lenguaje de signos forman monemas, unidades dotadas de contenido significativo. El LSE se sirve de seis parámetros formativos quinésicos: el queirema o configuración de la mano (los dedos extendidos en forma de estrella, el puño cerrado, etc.), el 
toponema o lugar de articulación (delante del torso o de la cara, etc.), el kinema o movimiento de la mano (recto, en forma de arco, etc.), el kineprosema o dirección del movimiento de la mano (hacia abajo, hacia la derecha, etc.), el queirotropema u orientación de la mano (con la palma hacia arriba, hacia el oyente, etc.) y el prosoponema o expresión de la cara. El cambio de cualquiera de estos parámetros cambiará el contenido del signo: si signamos TRABAJO y cambiamos únicamente la configuración de la mano (extendiendo los dedos), un sordo interpretará PLACER.

El LSE no distingue categorías funcionales, de modo que el mismo signo gestual representa los contenidos CURIOSO-CURIOSIDAD-CURIOSEAR o DEBILIDAD-DÉBIL-DÉBILMENTE. A la inversa, en ocasiones un solo signo representa conceptos que las lenguas habladas diferencian léxicamente: es el caso de AQUÍ, HOY y ESTO, todos los cuales guardan relación con el presente.

La autora divide los signos en tres grupos según el grado de iconicidad: 1) los signos motivados serán icónicos o deícticos, 2) signos intermedios y 3) signos arbitrarios. En el primer grupo tiene cabida BURGOS (las dos manos forman sendas agujas para representar las torres de la catedral) $o$ MUJER (el pulgar y dedo índice de la mano derecha se agarran al lóbulo de la oreja derecha como si se tratara de un pendiente); las personas gramaticales (señaladas con el dedo índice) y el tiempo cronológico (pasado, presente, futuro) son ejemplos de signos deícticos.

Los signos intermedios son aquellos que se basan en el alfabeto manual $-s i, y, o-o$ en otros signos del entorno visual (el signo de las ciudades y provincias españolas procede de las siglas de las matrículas de los coches).

El tercer grupo de signos arbitrarios incluye los de origen desconocido, que con toda probabilidad no podrán ser descifrados por una persona que desconozca el código.

La estrecha relación entre iconicidad y cultura queda patente de una manera especial en los dos primeros signos: el signo gestual correspondiente a MUJER no sería válido en una sociedad en la que no existiera la costumbre de poner pendientes a las niñas recién nacidas o en la cual la mujer no acostumbrara llevar este tipo de adorno. Otro ejemplo interesante de cómo pueden variar los signos según el entorno geográfico se encuentra en PERRO: en Valladolid y Madrid el signo gestual representa el hocico del animal, mientras que en Barcelona se unen las palmas de ambas manos, tal vez para representar la boca que se cierra. Como todavía cabrían otras interpretaciones y por lo tanto otros signos gestuales, hay que preguntarse por los límites entre iconicidad y arbitrariedad.

Cuando se propone identificar la tipología del LSE, la autora descubre que determinados esquemas morfológicos podrían considerarse flexiones si 
su uso fuera sistemático. Como esto no se cumple, opta por adscribirlo a la tipología de las lenguas aislantes aunque, dado el carácter simultáneo del signo gestual frente a la secuencialidad de las lenguas habladas (sometidas al tiempo), habría que modificar la definición de lengua aislante. La expresión del concepto de /"plural"/ es un claro ejemplo de por qué es difícil hablar de lengua flexiva en el caso del LSE. En principio hay tres maneras de pluralizar un signo gestual: 1) mediante la repetición del signo; 2) a través del signo MUCHO seguido del signo que se desea expresar en plural, repetido; y 3) signar con ambas manos, lo que denota gran expresividad. No tenemos más datos sobre el uso de las dos primeras posibilidades, pero en los textos que incluye la autora no es infrecuente encontrar tanto el signo MUCHO como la repetición del signo.

Si quisiéramos basar el estudio de las regulaciones del discurso gestual en lo que sabemos de la morfología de las lenguas naturales, podríamos encontrar muchas semejanzas, como por ejemplo el género, el número, el aspecto o el tiempo. No obstante, esto no quiere decir que exista un paralelismo total o que funcionen de la misma manera. Por ejemplo, ÉL tiene dos signos diferentes, según la persona esté presente en el momento de la conversación o no. El lenguaje gestual carece de un artículo determinado tal y como existe en español; sin embargo, esto de ninguna manera significa que el LSE no tiene un medio para designar un ser o un grupo concreto. De la misma manera que los lingüistas modernos critican a los gramáticos antiguos porque intentaban ajustar la gramática de las lenguas romances a los moldes del latín, tampoco podemos basar el estudio del LSE en lo que sabemos de otras lenguas: sería mejor describir el LSE y luego comparar la estructura del LSE con la gramática del español. Parece haber una cierta tendencia a mezclar ambos procedimientos, lo que resta claridad a un estudio tan importante como novedoso.

Se descubren algunas reglas de sintaxis. El orden de palabras preferido parece ser [Núcleo + Adyacente], ya se trate de un sintagma nominal [sustantivo + adjetivo] o [cláusula + negación]. El lugar de relieve es al principio o al final del enunciado.

La sintaxis del LSE es directa en el sentido de que el sujeto gramatical coincide siempre con el sujeto lógico:

\section{YO-GUSTAR-EXACTAMENTE-F.P. $\rightarrow$ «Me gusta la formación profesional»}

El LSE rehúye palabras «vacías» o gramaticales y potencia la yuxtaposición $y$, se supone, algún tipo de "pausa" para marcar las fronteras. No existen signos gestuales para ser y estar ni para tener.

PASADO-CELIA-TRISTE $\rightarrow$ «Celia se puso triste» 
Cuando se precisa un marcador copulativo, los sordos emplean el signo que corresponde a SE LLAMA:

$$
\text { «Es un hombre petulante» } \rightarrow
$$

ESTE-HOMBRE-SE LLAMA-PETULANTE

La existencia de una «homonimia visual parcial» también repercute en la sintaxis. El signo gestual que consiste en tomar el pulso representa igualmente MÉDICO y ENFERMO, con lo que podría haber ambigüedad entre "Es médico» $\mathrm{y}$ «Está enfermo». Esto se soluciona al insertar el signo TRABAJAR entre los otros dos:

\section{ÉL-TRABAJAR-MÉDICO $\rightarrow$ «Es médico»}

Ya hemos citado el papel que juega la lógica en el lenguaje gestual. También es importante para los fenómenos de coordinación y subordinación. Muchos estructuras que se consideran subordinadas se basan en variantes de las premisas lógicas de CAUSA y EFECTO o RESULTADO. Mientras que las lenguas naturales tienen un elenco de marcadores para indicar los distintos contenidos, de modo que el orden de las cláusulas no afecta la interpretación del mensaje, en el lenguaje gestual el orden lógico es preceptivo, incluso cuando el signo CAUSA encabeza el enunciado. Un grupo de niños sordos no entendía la siguiente oración de uno de sus libros de texto:

«la vegetación depende de dos factores: suelo y clima»

hasta que se lo glosaron en lenguaje gestual:

CAUSA-LLUVIA-Y-SOL-TIERRA-BIEN-PLANTAS-BIEN-(pausa)-
CAUSA-LLOVER-NO-TIERRA-SECA-PLANTAS-MAL, o

TIEMPO (atmosférico)-Y-TIERRA-MUY-IMPORTANTES-PARAPLANTAS-BIEN-O-MAL

Sin embargo, este orden de palabras no es obligatorio en oraciones condicionales.

También es interesante resaltar la relación que existe entre determinadas estructuras y contenidos. En el LSE las adversativas restrictivas y las concesivas tienen la misma estructura, que comparten además con las comparativas de desigualdad, mientras que la adversativa excluyente (con sino) se expresa con una estructura yuxtapuesta. 
La gramática tradicional puso el techo de la lingüística en la oración y allí terminaban sus análisis. Ahora bien, si el lenguaje es un medio de comunicación, habría que partir del mensaje. Si no cabe hablar de partes del discurso en el lenguaje gestual, también es difícil etiquetar las funciones oracionales (sujeto, objeto directo, etc.). M. ${ }^{a}$ Á. Rodríguez, después de analizar numerosas grabaciones, prefiere estudiar el discurso mediante el análisis del tema y el rema. Así también soslaya la necesidad de segmentar el discurso en «oraciones.»

La viabilidad de este tipo de análisis se hace patente cuando se intenta descubrir el orden de palabras en el lenguaje gestual. Los numerosos estudios no aportan datos concluyentes, aunque casi todos afirman la importancia de la topicalización, que guarda una estrecha relación con la expresividad, lo que la autora considera el componente más importante del lenguaje de signos.

Este excelente estudio viene a llenar un vacío existente en la lingüística española. Como es el caso de muchos estudios pioneros, los problemas que plantea pueden llegar a oscurecer su aportación a la ciencia. La importancia de este libro es tripartita. En primer lugar, llama nuestra atención sobre la situación de los sordomudos: en este caso las buenas intenciones han sido insuficientes. El lenguaje gestual es el medio de comunicación connatural a los sordos; como sistema de comunicación se presta a un estudio lingüístico, con el fin de identificar los mecanismos que lo rigen. En segundo lugar, cada lengua tiene su propio sistema, al que hay que describir desde dentro. Toda ciencia procede desde lo conocido hacia lo desconocido: ahora que contamos con una descripción del LSE, se prestará a un estudio inmanente, ajeno a la búsqueda consciente o inconsciente de semejanzas con las lenguas orales. Esto significa que la "gramática» del LSE no estará organizada de la misma manera que la gramática de la lengua española, y gran parte de las observaciones que hace $M^{a}{ }^{a}$ Á. Rodríguez apuntan en esta dirección. Finalmente, el lenguage es, por encima de todo, un sistema de comunicación; el cotejo de la organización de la información y la relación entre estructuras y contenidos en el lenguaje gestual y las lenguas naturales tiene un interés extraordinario. 